\title{
FELICIDAD Y PENSAMIENTO EN EL RENACIMIENTO ESPAÑOL: EL CASO DE LA NUEVA FILOSOFÍA DE LA NATURALEZA HUMANA (1587)
}

\author{
THE CONCEPT OF HAPPINESS AND THOUGHT IN SPANISH \\ RENAISSANCE: THE CASE OF NUEVA FILOSOFÍA \\ DE LA NATURALEZA HUMANA (1587)
}

\author{
Santiago ARROYO SERRANO \\ Universidad de Salamanca \\ sarroyo@usal.es
}

\begin{abstract}
Resumen: En este artículo analizamos la importancia de la felicidad en el Renacimiento español y especialmente en Nueva filosofía de la naturaleza humana (1587), considerado uno de los tratados filosóficos más importantes del Renacimiento español que vincula de manera directa los estados de ánimo y la felicidad con la salud. En esta obra se recupera la tradición que sostiene cómo a través del ejercicio de la filosofía se puede alcanzar la felicidad, tema relevante en el humanismo español, que al mismo tiempo recobra algunas de las tradiciones clásicas.
\end{abstract}

Palabras clave: Felicidad, Pensamiento, Renacimiento, Tradición, España.

\begin{abstract}
In this article we analyse the importance of the concept of happiness in the Spanish Renaissance and especially in Nueva filosofia de la naturaleza humana (1587), considered one of the most important philosophical treatises of the Spanish Renaissance, as it links directly moods and happiness with health. In this work we recover the tradition that maintains how through the exercise of philosophy it is possible to achieve happiness, a relevant topic in Spanish humanism, which at the same time brings to the present some of the classical traditions.
\end{abstract}

Keywords: Happiness, Thought, Renaissance, Tradition, Spain. 


\section{La felicidad en el pensamiento: una introducción.}

En este artículo analizamos las implicaciones de la felicidad en una de las obras fundamentales del Renacimiento español, donde el carácter médico-filosófico de algunos autores aparece como una de las líneas temáticas esenciales, que se manifiesta como una de las tradiciones literarias en el pensamiento español, con un estudio sobre la naturaleza humana publicado en el año 1587 y que, a pesar de las numerosas polémicas suscitadas sobre la posible autoría, contiene un mensaje relevante que resume y se reapropia de las concepciones de la felicidad humana en la tradición literaria hasta el Renacimiento, con ecos posteriores.

La búsqueda de la felicidad es un tema que recorre todo el pensamiento filosófico español a través de la literatura, desde el primer filósofo hispanorromano, Séneca, hasta la filosofía española contemporánea. Los médicos-filósofos del siglo XVI, puestos en valor por el gran hispanista francés Alain Guy (Guy: 1985), realizaron la integración de lo mejor de la tradición grecolatina con los valores cristianos.

La felicidad es un asunto recurrente en la historia del pensamiento en general, con visiones de autores como Aristóteles, Séneca, San Agustín, etc. Este concepto ha formado parte de un planteamiento ético que busca conseguir el bien, y un estado en el que el ser humano se encuentra en armonía con el universo. Son muchos los autores que han vinculado la felicidad con la sabiduría, la virtud o la paz. En la literatura, cuando pensamos en la palabra «felicidad» nos retrotraemos a la tranquilidad, a la calma, a la lectura, al diálogo, entre otras personificaciones del significado que puede tener «ser feliz». Estamos ante un concepto abierto a la interpretación pero con un profundo componente de carácter práctico y que afecta a la mayor parte de las esferas de la existencia.

Aristóteles en la Ética a Nicómaco (Aristóteles: 1985) ya defendió el fin último del hombre como la búsqueda del bien, para encontrar en todo lo que hacemos el disfrute y la felicidad. Este fin último es, por lo tanto, la felicidad, y la tarea propia del hombre es la búsqueda del estado feliz, aunque los caminos para encontrarla requieren un esfuerzo. Respecto de la felicidad, una cuestión es definirla y otra alcanzarla. Cada persona será feliz cuando realice la actividad que le corresponde en el mundo. Entonces, ¿cuál es el verdadero fin del hombre? Aristóteles plantea que la vida feliz es vivir de acuerdo con la razón. Para el estagirita el verdaderamente feliz es el sabio, como después recuperaría San Agustín suponiendo que lo común es desear la felicidad.

Epicuro, escribía Sexto Empírico, «decía que la filosofía es una actividad que procura con discursos y razonamientos la vida feliz» (Empírico, 1981: XI, 169). Para Epicuro «la filosofía ha de procurar la felicidad» (Marías, 1987: 28).

Para Séneca, que define la vida feliz como el estado en el que el individuo está «conforme con su naturaleza; lo cual no puede suceder más que si, primero, el alma está sana y en constante posesión 
de su salud» (Séneca, 2013: 52). La tradición filosófica y literaria busca como fin último la felicidad en todo lo que el hombre se propone y realiza, persiguiendo la felicidad individual y social.

San Agustín, que ya integra las tradiciones clásicas con el pensamiento cristiano, consideraba que la virtud es el mejor medio para alcanzar la felicidad. «La vida de la beatitud y del reposo consiste para el hombre en la racionalidad armoniosa de toda actividad» (San Agustín, 1957: 1.20.31) o lo que es lo mismo, «nadie es feliz a menos que lo sea por medio del bien supremo, que es visto y captado en aquella verdad a la que llamamos sabiduría» (San Agustín, 1982: 2.13.35). En las Confesiones escribe que la felicidad es el gozo que procede de la verdad. Para San Agustín, que se acerca a la felicidad bajo criterios platónicos, aristotélicos o estoicos, los medios para alcanzar la felicidad se encuentran determinados por su fe cristiana.

Es más, la felicidad ha preocupado también a nivel político y social por la influencia de los autores precedentes, fruto probablemente de su vigencia en la historia de la literatura y el pensamiento. En la Declaración de Independencia de los Estados Unidos, en 1776, aparece el lema Life, liberty and the pursuit of happiness, en el segundo párrafo del primer artículo de dicha declaración: derechos inalienables de la vida, la libertad y la búsqueda de la felicidad. La felicidad se entendería en este punto como tener las principales necesidades cubiertas. En la primera carta constitucional republicana en Francia, en 1793, aparece también el concepto de felicidad. En España, las Cortes de Cádiz, en el artículo 13 de la constitución de 1812, hacen referencia a que «el objeto de Gobierno es la felicidad de la Nación, puesto que el fin de toda sociedad política no es otro que el bienestar del individuos que lo componen».

Un repaso global de este término desde los textos filosóficos lo encontramos en el Diccionario de filosofía de Ferrater Mora, que apunta una definición de «felicidad» en la entrada de «eudemomisno» en cuanto a las morales llamadas materiales o concretas, que consideran la felicidad,

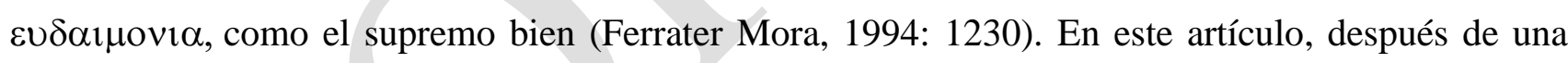
visión abierta de la felicidad desde la perspectiva de la literatura filosófica hispánica y algunas de las concepciones de felicidad en la historia del pensamiento, prestaremos atención a una obra que, fruto de la polémica suscitada en cuanto a su autoría, no ha sido detenidamente estudiada en cuanto a su valor literario e histórico y de la que, por lo tanto, aún quedan muchos análisis pendientes. Afirma Julián Marías, cuyo maestro Ortega también se interesó por el tema, que la felicidad pertenece a la vida humana (Marías: 1987). Este tratado analiza la felicidad desde este posicionamiento en cierto sentido vitalista.

Este tema es el que en el siglo XVI se abordó en la Nueva filosofía desde un punto de vista completamente innovador en cuanto a la perspectiva literaria, filosófica y humanística. Nos referimos a la Nueva filosofía de la naturaleza del hombre, no conocida ni alcanzada de los grandes filósofos antiguos: la cual mejora la vida y la salud humana. Compuesta por Doña Oliva Sabuco (Madrid, Pedro Madrigal 1587), un texto del siglo XVI que ha despertado gran interés por los historiadores del pensamiento en España, desde la primera historia de la filosofía española compuesta en lengua latina por Joseph Fernández Cuevas en el año 1858 en Madrid, con el título Historia Philosophiae ad usum 
academicae juventutis — cuyo libro 2, a partir de la página 56, se centra en la Historia Philosophiae Hispania-, pasando por Vives, Huarte y Fox Morcillo, que también estudian la filosofía de Oliva de Sabuco, para finalizar con Alain Guy, que fue el último historiador de la filosofía española que le dedicó un epígrafe a su pensamiento en dos de sus principales obras, Les philosophes espagnoles d'hier et d'aujourd'hui (1956) e Historia de la filosofía española (1985).

En este ensayo, considerado también por diversos investigadores como el primer tratado de medicina psicosomática, Sabuco realiza un análisis exhaustivo de las pasiones humanas en el que analiza cómo los factores mentales, sociales y literarios afectan a la salud corporal del ser humano, y especialmente a su felicidad. En este sentido, al final afirma que algunos autores han visto su obra como «un tratado moral, que garantice al hombre la siembre ansiada felicidad a través de un perfecto equilibrio entre cuerpo y alma en el estado de salud» (Díaz, 2003: 15).

Aquí pretendemos analizar este concepto filosófico, literario y moral en el contexto de un tratado de filosofía del Renacimiento español en el cual se resumen los valores fundamentales, de origen aristotélico, añadiendo un importante matiz y conectando la felicidad con la salud del cuerpo, siguiendo a Séneca, ya que, de este modo, se recupera la tradición clásica y al mismo tiempo se amplía, al conectar el bienestar mental y la felicidad con la salud humana y la literatura.

Debemos la recuperación global de esta obra para el hispanismo contemporáneo al pensamiento de Alain Guy (1956: 70-74). El gran filósofo hispanista afirmó que esta obra merece «mejorar la suerte y existencia cotidiana de su prójimo» (Guy, 1985: 141), después de «denunciar el fracaso de la medicina clásica en la curación de los males más comunes como las epidemias, afirma que casi todas las afecciones patológicas tienen como origen las preocupaciones» (1985: 142). ¿Es, por lo tanto, la filosofía o la literatura una herramienta para la salud y la felicidad del ser humano? Podemos afirmarlo en el planteamiento que subyace a lo largo de toda la obra, en el que la pensadora realiza una importante conexión entre felicidad y literatura. Es este punto donde se encuentra la verdadera innovación del texto publicado en 1587 ya que, sin saberlo, este planteamiento aparece como un principal antecedente de toda la psicoterapia actual a través de la insinuación retórica.

La Nueva filosofía es un canto a la búsqueda de la felicidad y las claves para alcanzarla, atendiendo a cuestiones corporales, sexuales, ambientales y sociales para obtener la alegría. Dedica de manera concreta uno de los capítulos a la felicidad, que es el que analizaremos aquí. Un poema de Marcial nos recuerda el planteamiento de Sabuco (Conde Parrado, 2014: 99):

\footnotetext{
Estas son, querido Marcial, las cosas que hacer pueden la vida más feliz patrimonio heredado, no sufrido, un terreno no ingrato, calor siempre, pleitos no, escasa toga, alma serena, un vigor natural, un sano cuerpo, sabia humildad, amigos de tu rango, una mesa sin boato frugal dieta, noches, sin ebriedad, despreocupadas, el lecho no tedioso, pero casto, un sueño que haga breves las tinieblas; querer ser lo que eres: no anhelar más; ni ansiar ni tener el último día.
} 
Como decía Claudiano en su poema Feliz aquel en la traducción de Antonio Alvar (Cuenca, 1981: 149), «Feliz quien pasa su vida en los campos propios, —quien de niño ve la misma casa que de anciano-», u Horacio en las Epístolas: «Neve putes alium sapiente bonoque beatum» («No juzgues a nadie feliz sino al sabio y al bondadoso»; Morajelo, 2008: 1, 16, 20).

\section{Oliva de Sabuco o Miguel Sabuco: una figura controvertida.}

Es muy posible que la autora que firma esta obra sea la mujer más polémica del Renacimiento español y sobre la que más se ha escrito y estudiado en gran parte del mundo (no en su tierra natal ni en España, donde ha sido menos valorada, tal vez por su condición femenina). Nació el 2 de septiembre de 1562 en Alcaraz (Albacete) y fue la quinta hija del matrimonio formado por Miguel de Sabuco —médico a quien se atribuyó la autoría de su obra después de su publicación, en tiempo muy reciente- y su primera esposa, Francisca de Cózar. Su educación, que tuvo lugar dentro de su ciudad natal, gran núcleo del saber en lo que hoy es Castilla-La Mancha, debió correr en primer lugar a cargo de su padre, que más adelante, además de su maestro fue su colega, y después del licenciado Gutiérrez, hasta que en 1579 disfrutó de las enseñanzas del humanista Pedro Simón Abril, también albacetense; y también es probable que tuviera algún contacto con el convento franciscano en el que existían cátedras de filosofía y de teología. A este magisterio, que pudiéramos llamar el regular, hay que sumar el de los muchos y cultos amigos de su padre, de cuyas tertulias y coloquios obtendría continuos beneficios intelectuales (Arroyo, 2007: 207).

Siendo aún muy joven, el 18 de diciembre de 1580, Oliva contrajo matrimonio con Acacio de Buedo, lo que no le impidió continuar colaborando con su padre al menos hasta 1587, fecha de la publicación de la Nueva filosofía de la naturaleza humana, de la que, en su portada y carta dedicatoria al rey, se declara autora. Parece que aquel matrimonio suyo no tuvo descendencia y, aunque se ha insinuado que los cónyuges en algún momento pudieron alejarse de Alcaraz para sustraerse de las habladurías que a buen seguro debió de producir la recogida por parte de la Inquisición de la segunda edición de sus obras, lo más probable es que continuaran viviendo allí, donde el marido desempeñó varios cargos públicos, ostentando el de corregidor en 1622, año en el que con toda probabilidad falleció. La obra de Oliva de Sabuco, publicada en 1988 en Madrid por Ricardo Fe (Díaz, 2003: 198), sigue hoy sometida a una gran polémica sobre su supuesta autoría.

Sin embargo, en la Nueva Filosofía sobre la naturaleza humana nos encontramos el pensamiento, en el que se descubre una sensibilidad característica - que consideramos propia del lo femenino - de una mujer que llevó a cabo una filosofía de las pasiones, en la que el cuerpo como materia de los afectos humanos juega un papel fundamental. En la obra hace un recorrido por las distintas pasiones del hombre, como el enojo, la tristeza, el miedo, el amor, el deseo, el placer, la alegría, la amistad, la desconfianza, la felicidad, el odio, la vergüenza, la misericordia, la lujuria, los celos, la venganza, el sufrimiento, etc., todas ellas con sus remedios y puestas de relieve desde una perspectiva psicosomática para mejorar la salud humana, poniendo las bases de lo que hoy es la psicoterapia o un ámbito de la psicología humanista, en aquel momento abordado desde la filosofía y 
en forma de diálogo entre tres pastores — como otras obras fundamentales de la filosofía del Renacimiento español一, basada en la tradición literaria grecolatina.

El cuerpo es uno de los temas centrales de su obra, donde en algún momento materia y razón son difícilmente distinguibles. Habla de los dolores y heridas del cuerpo, de cómo le afectan el frío y del calor, incluso de la necesidad de descanso después de la comida principal, todo ello unido a las pasiones del alma, que analiza minuciosamente. Una de ellas, como veremos, es la felicidad, a la que dedicaremos el siguiente epígrafe.

\section{La concepción de la felicidad en la Nueva Filosofía.}

Este libro apareció «para dar luz de la verdad al mundo, y para que los venideros gocen de filosofía, y de la alegría y contento que consigo tiene» (Sabuco, 2009: 247). En este artículo explicaremos cómo concibe la felicidad en el sentido global del término y realiza una fisiología de las pasiones que afectan a la felicidad del hombre, de las relaciones con otros seres humanos. Además, señalaremos el hecho de que esta obra enlaza con autores actuales que pueden estar vinculados a esta corriente psicosomática y fenomenológica de lo corporal, hasta llegar a La terapia del deseo de Martha C. Nussbaum.

Nuestra aportación, además de hacer que se conozca un trabajo hasta hoy olvidado y en algunos casos marginal como es la Nueva filosofía de la naturaleza humana, pone de manifiesto a una de las figuras más importantes de la historia del pensamiento español, cuya obra aún no se ha estudiado a fondo -aunque, paradójicamente se le está concediendo gran valor en centros de estudio y universidades extranjeras-, la riqueza y profundidad de los temas que trata, así como los autores referenciados y comentados. En el área de la literatura filosófica hecha en España, la investigación actual tiene mucho que explorar y descubrir en los estantes de la bibliotecas y en el pensamiento de autores que merecen ser explicados para un mejor conocimiento de nuestra cultura y, por consiguiente, de nuestro pasado filosófico.

Nos apoyamos en un análisis de esta obra poco conocido, el del francés Alain Guy, filósofo hispanista que en sus cursos en la Universidad, en sus artículos, conferencias y obras, siempre se ha complacido en poner en valor - a veces incluso en exhumar y descubrir - a muchos pensadores «a contracorriente de las ideas recibidas en el extenso y atractivo linaje de numerosos heterodoxos españoles» (Guy, 1979: 302). La preocupación principal de Guy durante toda su vida de investigador fue demostrar el error histórico y la calumnia de la leyenda negra que pretendía que el pensamiento hispánico fuera acaparado por el monolitismo de la tradición y el conservadurismo. En cambio, ha subrayado sin cesar y con toda la razón, la diversidad de las corrientes de pensamiento, el pluralismo de las diferencias y las luchas de ideas. Ha rectificado así el sentido y le ha conferido justicia, objetividad y valor (Gabaude, 2005: 35-53).

Alain Guy destaca y percibe un profundo carácter práctico de la filosofía que aparece en la literatura de diferentes filósofos médicos del Renacimiento, como Huarte o Fox Morcillo, y que 
emerge incluso la tradición en el siglo XX con autores como Laín Entralgo, Gregorio Marañón y Carlos Castilla del Pino, por citar algunos.

Gracias al profesor Guy, embajador y gran especialista global del pensamiento ibérico, podemos conocer igualmente esta pléyade de filósofos-médicos españoles, de los que destaca su papel innovador y esclarecedor en diversos títulos. Uno de ellos es Francisco Valles (1524-1592), llamado el divino, médico atraído por Felipe II que expone en su libro en latín De sacra philosophia (1587) una doctrina ecléctica que, junto con Aristóteles, se inspira más en Boecio, Hugues y Richard de Saint-Víctor, (Guy: 1985). Entre estos pensadores también reconoce y pone en valor la obra de Oliva de Sabuco, erasmista, empirista, inconformista, lejana precursora de la psicoterapia (la salud a través de la filosofía) y de la medicina psicosomática, en la que se produce un debate entre espiritualismo y materialismo, que luego seguirían otros autores como Luis de Granada o Diego de Estella.

Según Guy, en un artículo publicado en la Encyclopédie Philosophique Universelle (1998: 455526), Sabuco recuerda a Raspail y constituye una gran escuela española del Siglo de Oro independiente. La obra muestra que todas las enfermedades provienen de una causa psíquica, la preocupación o enfado, por lo que defiende que para restaurar nuestra salud es indispensable restablecer en nuestra alma el buen humor. Toda una guía espiritual es entonces promulgada en función de una peculiar teoría de flujo vital en la que el juego alternado del «crecimiento» y del «decrecimiento» ordena todo el organismo. Antigalienista y antihipocrática, Sabuco exige la reforma de la medicina y se opone a la tradición; de espíritu religioso, se la siente animada por un erasmismo que reclama del resto medidas sociales de salud pública (contra los mayorazgos, los procesos, los duelos o la desigualdades); es, incluso, pionera en algunos aspectos de la ecología.

$\mathrm{Al}$ analizar la Nueva filosofía, encontramos elementos tradicionales y sus propias innovaciones, pero sobre todo aspectos esenciales relacionados con la felicidad mundana y literaria. ¿Qué significa la felicidad y cómo aparece en su obra en forma de diálogo? Es, como ya planteó Aristóteles, el fin último del hombre:

Veronio. Si vos pedís eso, señor Rodonio, yo pido otra cosa, y es que me declaréis aquel dicho escrito con letras de oro en el templo de Apolo; Nosce te ipsum. Conócete a ti mismo, pues los antiguos no dieron doctrina para ello, sino solo el precepto, y es cosa que tanto monta conocerse el hombre y saber en qué difiere de los brutos animales. Porque yo veo en mí que no me entiendo, ni me conozco á mi mismo ni á las cosas de mi naturaleza: y también deseo saber, cómo viviré feliz en este mundo (Sabuco, 2009: 88).

En el título XXII sobre los Afectos que dan salud y sustentan la vida humana, expone Sabuco que «hay otros afectos en el hombre que le dan y acarrean salud y vida (al contrario de los dichos) como son las dos columnas o empentas espirituales, que son esperanza de bien, alegría y contento» (Sabuco, 2009: 107). La denominación precisa de la felicidad en su obra es «esperanza de bien, alegría y contento» (Sabuco, 2009: 117). Aquí la felicidad adquiere un componente futuro, de proyecto:

Pues estos dos afectos principales y continuos de la cámara deste príncipe, que dan vida y cremento al cerebro del hombre por la concordia y amistad del alma que allí mora con las especies que allí entran, no habiendo ninguna contraria, desechada ni aborrecida, consérvase la amistad de alma y cuerpo, y crece y se aumenta lo corporal, que es la médula del cerebro y su jugo (Sabuco, 2009: 117). 


\section{Tropelías. Revista de Teoría de la Literatura y Literatura Comparada, 30 (2018)}

Felicidad y pensamiento en el Renacimiento español: el caso de la Nueva Filosofía...

En el epígrafe XXVIII titulado De la amistad y buena conversación, necesaria a la vida humana, como elementos para la felicidad, siendo la amistad y la buena conversación

[...] muy necesaria para la salud del hombre, porque el hombre es animal sociable, quiere y ama la conversación de su semejante, en tanto que algunos llamaron a la buena conversación, quinto elemento, con que vive el hombre, es necesario el hablar y conversar al ánima a sus tiempos y entender en algo de pasatiempo, porque el alma empleada y atenta en algo aprovecha para la salud, y al contrario estando queda y ociosa como el agua encharcada se pudre (Sabuco, 2009: 126).

En este caso vincula la amistad a la felicidad pues «también por otra razón son necesarios los amigos, porque si el alma no tiene en qué emplear su amor natural que brota para fuera ni con qué llenar sus deseos y gran capacidad, la cual se llena con lo amado, luego se marchita y desmaya, y hace melancolía y tristeza, quedándose como vacía y frustrado su apetito, deseo y acción natural» (Sabuco, 2009: 126). Y es que en la Nueva filosofía la amistad nos lleva a la salud, «el amigo es otro yo, y así el ser es la mayor felicidad y dejar de ser la mayor miseria, así es gran felicidad ser hombre dos veces teniendo amigo verdadero. Con el buen amigo los bienes comunicados crecen, y se hacen mayores, y los males y congojas se alivian y se hacen menores» (Sabuco, 2009: 126). Esta trascendencia o multiplicación en quien como «amigo procura las cosas del amigo como las suyas. Guarda el secreto y con el han de ser comunes los secretos del alma, y también las riquezas corporales. Todo lo de los amigos ha de ser común» (Sabuco, 2009: 126).

El título LVI, De los ornatos del ánima, nos conduce a la máxima socrática del «conócete a ti mismo» que recorre toda la obra, pues «otras cosas hay en el hombre que son unas hermosuras y ornatos del ánima, los cuales llamaron virtudes morales, las cuales son muy necesarias para el conocimiento de sí mismo, y para alcanzar la felicidad o bienaventuranza que puede haber en este mundo» (Sabuco, 2009: 155). Las resume en «cuatro principales, que son: Templanza, Fortaleza, Justicia, Prudencia. De las cuales nacen otras como magnanimitas, liberalitas, amicitia, gratitud, etc.» (Sabuco 2009: 155).

Ese conocimiento de sí mismo garantiza la plenitud y el mejor complemento para el alma, en el título LX, De la sapiencia, que es el mayor ornato del ánima:

La sapiencia es una ciencia de las cosas divinas y naturales, y conocimiento de las causas de todas las cosas: es una virtud y ornato, en el hombre la más alta y divina de todas, y que a todas las perfecciona; a esta trujo pegada consigo el ánima del cielo, tiene un sabor y olor de Dios, está perfecta en sólo Dios, y de allí le mana el ánima del hombre que él sólo la tiene; da gran contento y alegría, y por eso salud; es la cosa más amable que hay en este mundo, y todo hombre desea saber. Si la sapiencia tuviera forma visible, no hubiera cosa más amada de los hombres. Esta hace dichosos y felices en este mundo, y sin ella no hay felicidad (Sabuco, 2009: 163).

Es en el título LXI, que lleva por título De la felicidad que puede haber en este mundo, donde profundiza en el concepto de felicidad y cómo se encuentra en el mundo. Reproducimos aquí la definición, por su riqueza de matices:

La felicidad (que dice bienaventuranza), la que en este mundo de destierro puede haber, es un placer y alegría del alma que da gran salud al hombre, porque es una de las tres columnas que sustentan la vida humana: consiste en la sapiencia y conocimiento de las causas, y en obra del entendimiento, contemplando y atendiendo todas las cosas de este mundo como son, y en la elección de la prudencia, sabiendo tomar el 
medio en todas las cosas, el cual medio hace felice y dichoso al hombre, obrando las virtudes (que es el medio entre dos vicios) con alegría de buena conciencia, y en los deleites tomando el medio necesario de todo bien para el sustento de la vida y no más (Sabuco, 2009: 165).

Sabuco incorpora de nuevo el tema de la felicidad en la tradición filosófica y literaria occidental. «Dijo Platón: "El prudente evita la miseria, no el rico". Y dijo: "No puede ser ninguno feliz sin que sea sabio y bueno". Y al contrario los malos son míseros y desdichados» (Sabuco, 2009: 166). Por lo que «esta felicidad ha de ser obra del entendimiento, razón y prudencia, en lo cual eres hombre y te diferencias de los otros animales que no lo tienen, y no en ningún género de deleites sensuales que en estos comunicas con los animales» (Sabuco, 2009: 166). Aquí muestra la felicidad como algo eminentemente humano y defiende que además

Es una alegría, contento y placer de gozar todos los bienes que se nombran bienes de este mundo, de manera que al verdaderamente feliz no le han de faltar también los bienes temporales deste mundo necesarios; pero sabe que con muchas riquezas no puede ser feliz, porque traen consigo muchos males, como enojos, cuidados, hurtos, pleitos, y así no has de tener más de lo necesario a la vida. [...]

En un estado mediano sin mucha soberbia ni puntos vanos de honra, ni menos demasías en vanos de vanagloria, en vestidos, criados, ni comidas, que todo da gran fatiga y desasosiego, y quita la felicidad. Con sólo lo necesario a la vida, poniendo meta y raya, cada uno en su estado y proporción, puede ser felice, descojiendo el medio con la prudencia en todas las cosas; y tampoco puedes ser felice si no tienes alegría de buena conciencia, sirviendo y conociendo a Dios, porque sin esta todo es tristeza y congoja de espíritu. Y así te es necesario dejar los vicios y obrar virtudes, porque claro está que si no tienes la virtud temperancia, luego la demasía de la gula y la lujuria y vicios te quitará la felicidad, si no tomas el medio. Tampoco puedes ser felice si no tienes la virtud justicia, queriendo para el prójimo lo que quieres para ti, porque si no das a cada uno lo que es suyo luego has de andar en contiendas y pleitos, y en pecado (Sabuco, 2009: 166).

Como también dijo Fray Luís de León, para quien «la paz es a sus ojos el símbolo de la virtud y de la felicidad» (Guy, 1960: 168), o en reconocido poema, «Qué descansada vida, / es la del que huye del mundanal ruido, / y sigue la escondida senda por dó han ido / los pocos sabios que en el mundo han sido». Para él «la paz es el sumo de la felicidad» (Guy, 1960: 169).

Sigue Sabuco conectando la felicidad con la tranquilidad, la serenidad y el sosiego en la naturaleza, la prudencia y el término medio ya advertido como la virtud en la ética aristotélica, que en la tradición popular española se vincularía con la mayoría del refranero y dichos populares. La moderación, que tanta vigencia tiene hoy, desde la alimentación, el deporte o los placeres:

Díjolo Salomón, díjolo San Agustín, San Ambrosio, Boecio, Horacio, Séneca, Cicerón, Platón. Si todos los sabios cuantos lo han dicho, y lo han hecho, hubiéramos de referir aquí, fuera dar fastidio. Diocleciano, emperador de Roma, estando en el senado asentado en la silla imperial con la toga de emperador, se levantó y se quitó la toga, y la puso en la silla, y dijo al senado: «Señores, dadla a quien quisiéredes, que yo no la quiero», y se fue a una heredad y huerta que tenía apartada de Roma, y allí vivía en sosiego y quietud, y a los que le visitaban decía: «agora vivo, agora amanece para mí». [...]

Es mejor el sosiego y tranquilidad, y poca gente. Es mejor el poco dormir y levantar de mañana. Es mejor y más seguro estar flaco que gordo. Es mejor el poco comer que el mucho. Al rico le pesa porque se harta, y al pobre le place. Pues esto es así que nunca te has de hartar de riquezas, más vale no empezar y evitarás tantos daños como traen consigo, y vivirás en sosiego, felicidad y alegría verdadera, con la buena conciencia, y serás felice como Psophidio en esta vida, y con los santos que se contentaron con la pobreza en la otra, y no te darán muerte violenta en medio de la edad, como comúnmente por ellas el enemigo del género humano la acarrea y da a los mortales, sino vivirás en sosiego, y pasarás felice todo el curso de tu vida, contento y alegre con lo necesario a la naturaleza, y llegarás a la muerte natural por vejez, y acabase el húmido radical, la cual no se siente y se pasa sin dolor, como lo afirma Platón (Sabuco, 2009: 169). 
Guy, desde un hispanismo filosófico riguroso, plantea que «por encima de todo, un aspecto de Sabuco debe ser subrayado. Es el cuidado moral que le anima. Se ha visto cómo invita a los hombres a reformar sus tendencias, a ejercer sobre ellas el "self-control" gracias al libre albedrío» (Guy, 1987: 122). Este análisis de Guy refleja la total pervivencia de la Nueva filosofía, a la que contextualiza en la historia de la filosofía de manera elocuente:

[...] un cierto estoicismo de voluntad se revela en su pensamiento. Al contrario del descuido (o «destape») naturalista y laxista de nuestra época, pretendiendo huir toda «represión» como alienante, Sabuco predica el gobierno de sí mismo, el dominio de nuestras pulsiones, la subordinación de nuestros deseos y de nuestros actos al Bien, a la Comunidad humana y a Dios (Guy, 1987: 122).

Para defender finalmente que su proyecto terapéutico

[...] es sobre todo, una ética; los lectores recuerdan a qué punto enaltece la generosidad y el rechazo de la prepotencia del dinero; en contra del individualismo egoísta de un pseudo-liberalismo permisivo, demasiado difundido y deletéreo, en nuestro siglo XX que se termina, que elogia cínicamente la búsqueda del provecho y de la voluptuosidad sin freno, Sabuco se inserta dentro de la tradición ascética del cristianismo (Guy, 1987: 123).

Es por eso, de otra parte, por lo que José Luis Abellán ha percibido muy agudamente que «el pensamiento español se caracteriza por el énfasis puesto en la conciencia moral, lo que a su vez producirá una inevitable tendencia al idealismo de carácter ético» (Abellán, 1985: 2). Y al mismo tiempo sostiene Guy que

[e]sta filosofía del pensador de Alcaraz, ecléctica en la buena acepción de la palabra, tiene los pies en el suelo, en lugar de perderse dentro del Wölkenkukusheim de las abstracciones y de los aficionados a las quintaesenciar; puede incluso ser calificada de positiva, aunque no de positivista; empirista de una manera superior, anuncia la «metafísica positiva» de Bergson, Jacques Chevalier, Teilhard de Chardin y Paul Chauchard. Tal vez, en su tiempo, Sabuco ha predicado en el desierto (Guy, 1987: 123).

\section{A modo de conclusión.}

Podemos llegar a una conclusión de lo anteriormente expuesto que tiene que ver con la importancia de la felicidad en los tratados más importantes de la filosofía del renacimiento en España donde según han mostrado autores como Guy la filosofía siempre ha conocido una gran actividad, colocando precisamente su momento de mayor esplendor en el Siglo de Oro (siglos XVI-XVII), en el momento de la hegemonía hispánica. Después de cierta decadencia desde finales del siglo XVII hasta el siglo XIX, retoma su desarrollo en el siglo XX con un solo eclipse, el del periodo franquista. Según el hispanista francés se pueden distinguir en esta larga historia cinco constantes: el gusto por la lógica, la preocupación moral y política, la familiaridad con la medicina, la nostalgia y el sentido de absoluto.

Como viene marcando la actualidad son cada vez más los estudios que recuperan a autores clásicos y de la época moderna y sus aportaciones a la felicidad del tiempo presente. La aparición habitual en el seno de tradición filosófica en España de una experiencia médica y psiquiátrica, ¿es legado de terapeutas judíos o árabes que fueron los encargados de la salud pública peninsular? El propio Maimónides fue un ilustre médico-filósofo judío de Córdoba. Más adelante, en el siglo XVI, otros médicos filósofos como Gómez Pereira, y su Antoniana Margarita (1554), donde defiende un empirismo integral. Más adelante 
Juan Huarte de San Juan (1529-1588), en su Examen de los ingenios para las ciencias (1575), impulsa la filosofía natural y psicológica con el objetivo de reformar la pedagogía a través de un estudio científico de temperamentos que fundan los diversos tipos de carácter o ingenio. En este sentido se sitúa aquí Oliva de Sabuco, en la Nueva filosofía de la naturaleza humana defensora de que las enfermedades provienen de lo físico y lo psíquico, ya que para gozar de buena salud es indispensable reestablecer en nuestra alma el buen humor, por lo que hace al hombre responsable de su felicidad, como afirma en una de las máximas al final de su libro: «Tu te haces enfermo y valetudinario, tu te puedes hacer sano y fuerte. Ningún enemigo es más dañino para ti que tu mismo» (Sabuco, 2009: 415).

\section{Referencias bibliográficas}

ABELlÁn, José Luis (1985): «Utopía en el pensamiento español», Cultura, História e Filosofia, IV, pp. 7-19.

San Agustín (1957): «Génesis contra los maniqueos», en Obras de San Agustín. Vol. XV. Madrid, Biblioteca de Autores Cristianos, pp. 495-569.

_ (1982): Del libre albedrío. Madrid, Biblioteca de Autores Cristianos.

ARISTÓteles (1985): Ética Nicomaquea. Trad. Julio Pallí Bonet. Madrid, Gredos.

Arroyo, Santiago (2007): Diccionario de Pensadores de Castilla-La Mancha. Toledo, Junta de Comunidades de Castilla-La Mancha.

Conde PARrado, Pedro, ed. y trad. (2014): Marco Valerio Marcial, Antología de epigramas. Gijón, Trea.

CuencA, Luis Alberto - Alvar, Antonio, comps. y trads. (1981): Antología de la poesía latina. Madrid, Alianza.

DíAz DíAZ, Gonzalo (2003): Hombres y documentos de la Filosofía Española. Madrid, CSIC.

EMPírico, Sexto (1981): Contra los profesores. Trad. Jorge Bergua Cavero. Madrid, Gredos.

FERnÁndez Cuevas, Joseph (1858): Historia Philosophiae ad usum academicae juventutis. Madrid, Eusebio Aguado.

FERrATER MORA, José (1994): Diccionario de filosofía. Barcelona, Ariel.

GABAUdE, Jean Marc (2005): «Situación de la obra de Alain Guy», en José María RoMERo BARÓ, coord., Homenaje a Alain Guy. Barcelona, Universidad de Barcelona, pp. 35-53.

GUY, Alain (1956): Les philosophes espagnoles d'hier et d'aujourd'hui. Toulouse, Privat. (1979): «Modernité du philosophe Sabuco», en AA. VV., Les cultures ibériques en devenir, essais publiés en hommage à la mémoire de Marcel Bataillon. París, Fondation Singer-Polignac, pp. 297-309.

(1985): Historia de la filosofía española. Barcelona, Anthropos.

- (1987): «Miguel Sabuco, psicólogo de las pasiones y precursor de la medicina psicosomática», Al-Basit. Revista de Estudios Albacetenses, 22, pp. 111-123. 
(1998): «La philosophie en Espagne», en Jean-François MATTEI, dir., Encyclopédie Philosophique Universelle. Le discours philosophique. Vol. IV, Le discours philosophique, pp. 455-526. París, PUF.

MARÍAS, Julián (1987): La felicidad humana. Madrid, Alianza Editorial.

MorAJElo, José Luis, ed. (2008): Horacio, Epístolas. Madrid. Gredos.

Nussbaum, Martha C. (2003): La terapia del deseo: teoría y práctica de la ética helenista. Trad.

Miguel Candel. Barcelona, Paidós.

Sabuco, Miguel (2009): Nueva filosofía. Ed. crítica de Samuel García Rubio y Domingo Henares.

Albacete, Instituto de Estudios Albacetenses «Don Juan Manuel».

SÉnECA (2013): Sobre la Felicidad. Versión y comentarios de Julián Marías. Madrid, Alianza. 